\title{
Evidence for strategic egg production in a hermaphroditic cestode
}

\author{
C. WEDEKIND*, D. STRAHM and L. SCH ÄRER \\ Abteilung Verhaltensökologie, Zoologisches Institut, Universität Bern, CH-3032 Hinterkappelen, Switzerland
}

(Received 7 February 1998 ; revised 16 April 1998; accepted 16 April 1998)

\section{SUMMARY}

The cestode Schistocephalus solidus is a simultaneous hermaphrodite that grows in 2 intermediate hosts and reproduces rapidly within a few days in the gut of a bird. Reproduction takes place by self- or cross-fertilization. Here, it was tested whether egg production differs between $S$. solidus that reproduce alone and those that are allowed to reproduce in pairs. Egg production in an in vitro system was found to depend on the cestodes' social situation. When kept alone, larger cestodes produced larger eggs. This was not so when kept in pairs - the difference between these 2 reproductive modes being highly significant in this respect. Further experiments revealed that, within the first 3 days, these hermaphrodites produced a larger total egg mass when kept alone than when kept in pairs. This was also reflected by the energy contents of the cestodes after this time-span: selfers had used up more energy than paired worms. Furthermore, S. solidus appeared to adjust its investment per egg depending on whether the offspring will be the result of self- or cross-fertilization. Selfers produced larger numbers of eggs, but these eggs were smaller and contained even smaller embryos per given egg size than eggs of potentially outbreeding cestodes. Selfed eggs reached lower hatching rates. Although this is to be expected from inbreeding depression it may also be an effect of the reduced maternal investment per egg. The observed phenotypic plasticity in the reproduction of $S$. solidus is discussed within 4 evolutionary frameworks: local mate competition adjusted for hermaphrodites, the hermaphrodite's dilemma, bet-hedging, and sib-competition.

Key words: Schistocephalus solidus, bet-hedging, sib-competition, selfing, outbreeding, egg size, life-history.

\section{INTRODUCTION}

Hermaphrodites possess functional male and female reproductive system(s) during at least part of their life. There are 2 major types: (1) sequential hermaphrodites who are able to change from one sex to the other during their life, e.g. certain corals, fish and polychaetes, and (2) simultaneous hermaphrodites who are male and female at the same time and usually use both functions in reproductive acts, e.g. many gastropods, flatworms etc. (see list in Michiels, 1998). Simultaneous hermaphrodites give rise to a number of biologically most interesting questions (e.g. Jarne \& Charlesworth, 1996; Michiels, 1998), e.g. sex allocation (Charlesworth \& Charlesworth, 1981; Charnov, 1982, 1996; Petersen, 1990a), the evolution and the consequences of selfing and outcrossing (Charlesworth \& Charlesworth, 1987; Jarne \& Charlesworth, 1993), or the hermaphrodite's dilemma, i.e. conflict and cooperation during copulation and sperm use (Axelrod \& Hamilton, 1981; Leonard, 1990).

Sex allocation and reproductive conflicts of simultaneous hermaphrodites have been extensively studied in plants (reviewed by Brunet, 1992). In animal studies, many papers concentrate on species

* Corresponding author. Tel: +3163191 11. Fax: + 31 63191 41. E-mail: wedekind@esh.unibe.ch with external fertilization (e.g. in fish: Fischer, 1981; Petersen, 1990b: Leonard, 1993; Mary, 1994; Petersen \& Fischer, 1996; in polycheates: Sella, 1990; Premoli, Sella \& Berra, 1996). However, most simultaneous hermaphrodites are species with internal fertilization (Michiels, 1998). Studies on reproductive conflicts in this group of hermaphrodites have been performed with, for example, a sea slug (Leonard \& Lukowiak, 1991), a barnacle (Raimondi \& Martin, 1991), snails (De Visser, Ter Maat \& Zonnefeld, 1994; Doums et al. 1996), flatworms (e.g. Vreys \& Michiels 1997), and a mussel (Johnston, Das \& Hoeh, 1998).

Most parasitic platyhelminthes are simultaneous hermaphrodites with internal fertilization that are, in contrast to many non-parasitic species, normally capable of self- and cross-fertilization (reviewed by Nollen, 1983). There is evidence for a number of parasitic helminths that they are not completely outbreeding when reproducing in pairs or in groups. Some of the sperm found in the receptaculum seminis are their own sperm (reviewed by Nollen, 1983 ; Trouvé et al. 1996; Nollen, 1997). Therefore, sperm competition (Parker, 1984) is likely to exist already in pairs of helminths.

Although a number of parasitic helminths can be kept in the laboratory under fairly controlled conditions, there are still very few studies that address the interesting problems of reproductive 
conflicts and strategies that arise from hermaphroditism in parasites (Trouvé et al. 1996; reviewed by Nollen, 1983 and Michiels, 1998). This is surprising since parasitic platyhelminthes offer the possibility to study offspring that derive from different mating situations, i.e. that are the result of potential reproductive strategies of their parents, in controlled infection. This allows measurement of important fitness parameters such as infectivity, growth and virulence of the offspring in their natural environment, which can then be related to the reproductive strategies of their parents. However, it is first of all necessary to test whether there exists a parasitic platyhelminth which is able both to react to different mating situations and to adjust its reproduction in a strategic manner. Here, the first observational data are presented and, based on them, the first experimental studies on the pseudophyllidean cestode Schistocephalus solidus that address the question of whether their cestode is able to reproduce strategically in response to given mating situations.

S. solidus is a simultaneous hermaphrodite that has 2 intermediate hosts and 1 final host (Clarke, 1954). The cestode matures in the gut of a fish-eating bird (definitive host). The eggs pass out into the water with the bird's faeces. After several weeks of development, the free-living larva, the coracidium, hatches and moves around for several hours during which time it has to be ingested by a cyclopoid copepod. Growth and development takes place in the body cavity of this first intermediate host, and, if the later is swallowed by a three-spined stickleback (Gasterosteus aculeatus), in the second intermediate host. The life-cycle is completed when the infected fish is swallowed by a bird. The cestode matures and reproduces within a few days in the bird's gut (Tierney \& Crompton, 1992).

The bird can be replaced by an in vitro system that allows observations and study of reproduction of this parasitic worm under controlled conditions. In this study it was tested (i) whether cestodes kept singly produce a different total egg mass within the first 3 days in the adult phase than cestodes which are kept in pairs, (ii) whether such a difference in produced egg mass is also reflected in the energy content of worm tissue after this time-span, (iii) whether reproductive parameters change over the time in these 2 mating regimes, (iv) whether the possibility of cross-fertilization has consequences on the parents' relative investment per egg, measured as egg size and embryo size, and (v) whether hatching rates differ between eggs of these 2 mating regimes.

\section{MATERIALS AND METHODS}

\section{Parasite culture}

Naturally infected sticklebacks were caught from closely connected ponds in Bochum (Germany), brought into our laboratory, kept at $8-12{ }^{\circ} \mathrm{C}$ in running water and fed with living Tubifex and thawed Artemia until removal of the plerocercoids.

The cestodes were cultured in vitro using a technique modified from Smyth (1954): plerocercoids were removed aseptically from sticklebacks, weighed to the nearest $\mathrm{mg}$, and inserted into seamless semipermeable tubes (Visking Dialysis Tubing 8/32, diameter: $6 \mathrm{~mm}$, about $5-10 \mathrm{~cm}$ in length). Either 1 or 2 cestodes were put into each tube. Whenever possible, cestodes that were put together in pairs originated from different fish but were of similar size. The tubes were suspended into 250 or $500 \mathrm{ml}$ bottles filled with sterilized culture medium based on Minimum Essential Medium with Earle's salts, L-glutamine, and 25 mM HEPES buffer (Sigma) and additives (per litre of medium: $1 \mathrm{~g}$ penicillin/ streptomycin, $6.5 \mathrm{~g}$ D-glucose), and titrated with $\mathrm{NaOH}$ to a $\mathrm{pH}$ of $7 \cdot 5$. These culture bottles were placed into a water bath at $40^{\circ} \mathrm{C}$ and shaken continuously with a horizontal motion throughout the normally 3 days of incubation. Only cultures where all cestodes were alive and still in the tube at the end of the incubation period were considered for further analyses (the few cultures with dead worms never contained any eggs; in a few cases a cestode managed to escape from the tube). At the end of the incubation period the cestodes were removed and the eggs collected by rinsing the inside of the tube with tap water into a Petri dish. After the eggs had settled the water above them was replaced with tap water to remove remaining culture medium and waste products of the adult cestodes.

To determine egg number, the eggs in a Petri dish were stirred up and allowed to settle in a way that a distribution of eggs as even as possible was achieved within the Petri dish. Then the Petri dish was placed under a microscope which was connected to a video system. The eggs could easily be counted from the screen (total magnification including screen: $\times 140$ ) which showed an area of $2.86 \mathrm{~mm}^{2}$ of the Petri dish. At least 15 such areas distributed as evenly as possible over the Petri dish were counted to get an estimate of the total number of eggs of a clutch. In some cases the method differed slightly (a binocular was used instead of a microscope, and the areas counted were $1.6 \mathrm{~mm}^{2}$ each).

To measure egg size, some randomly chosen eggs were put under the microscope and projected to a screen (total magnification including screen: $\times 1400$ ). From the screen, the maximal length and the maximal width of the intersection of the eggs was measured (this method allowed measurements to the nearest $\mu \mathrm{m}$ real size). Egg volumes were calculated with the formula of an ellipsoid (length $/ 2 \times$ $(\text { width } / 2)^{2} \times 4 \pi / 3$ ). In the non-experimental clutches 10 eggs were measured, in the experimental ones 15-30 eggs per Petri dish.

The data were analysed with systat (systat, 1992). Parametric statistics were used only when 
data plots had indicated that the assumptions of such statistics were not violated, otherwise non-parametric statistics were used.

\section{Non-experimental cultures}

A total of 62 cultures with 1 tube $/ 250 \mathrm{ml}$ culture bottle and $1(n=40)$ or $2(n=22)$ cestodes/tube were started during 2 years for other experiments (Wedekind \& Milinski, 1996; Wedekind, 1997). All these cultures were run for 3 days, and for all of them at least 10 randomly chosen eggs were measured; however, the eggs of a clutch were not counted.

\section{Experimental cultures}

A series of experimental cultures was started to test whether the observed differences reported in Fig. $1 \mathrm{~A}$ and $B$ could be replicated when potentially confounding variables were controlled for. Moreover, several new techniques were used to measure egg production in more detail, i.e. to test whether isolated worms differed from paired worms in egg size, egg number, embryo size, hatching rates, total egg mass, and energy loss after 3 days of incubation; and egg size, egg number and hatching rates over 5 days of incubation.

In the first series of experiments, bottles with $500 \mathrm{ml}$ of culture medium were used into which 2 separate tubes were suspended. One $S$. solidus larva freshly removed from an infected stickleback was put into one tube, 2 others into the other tube. Whenever possible, cestodes that were put in pairs originated from different fish. The cultures ran for 3 days. Egg number and size of at least 15 randomly chosen eggs measured at a time before hatching could occur were determined ( $n=32$ culture bottles).

After the experiments were terminated, the worms were briefly rinsed in distilled water and then placed into $5 \mathrm{ml}$ of glass scintillation vials with a plastic top and frozen at $-20^{\circ} \mathrm{C}$. Worm pairs were frozen jointly. Oxygen micro-bomb calorimetry (Phillipson, 1964) was performed on 43 samples (21 alone and 22 pairs, the mean weights of this subsample of the first experiment did not differ significantly; alone: $267 \cdot 2 \mathrm{mg} \pm 27 \cdot 6$ s.E. in pairs: $292 \cdot 6 \mathrm{mg} \pm 23 \cdot 3, \quad t=0 \cdot 71, \quad P>0 \cdot 4)$. Samples had been in the freezer for 3-11 months before they were freeze-dried in a Secfroid lyophilisator for $72 \mathrm{~h}$. The dried samples were homogenized with a mortar and pestle and, depending on the amount of material available, some of it or all was pelleted in a pellet press. The weights of these pellets ranged from $9 \cdot 4$ to $31.9 \mathrm{mg}$. These pellets were combusted in a modified Phillipson Oxygen Microbomb Calorimeter developed by Ingolf Lamprecht at the Institut für Biophysik, Freie Universität Berlin, Germany. Two combustion bombs that had been calibrated in this calorimeter with a reference substance of known caloric content (benzoic acid) were used. Both combustion bombs were used about equally often with samples of both treatments (2-tailed Fisher's Exact Text, $P>0 \cdot 7)$.

In a second experiment, nine $500 \mathrm{ml}$ culture bottles again with a 1 -worm and a 2 -worm culture each in separate tubes were used. In this experiment, however, the 9 culture bottles were started at the same time and run for 5 days. A conically shaped glass pipe with a small opening was attached at the bottom of each tube. This opening allowed the eggs to fall into a little collecting beaker which could be exchanged. This allowed collection of eggs/tube after the third, fourth and fifth day of culture. The eggs were kept in small Petri dishes in a climate chamber at $20^{\circ} \mathrm{C}$ and $12 \mathrm{~L} / 12 \mathrm{D}$. The number and size of these eggs were measured on the 15 th or 16 th day of incubation as explained above. S. solidus eggs normally hatch shortly after this time under the conditions we had set them (own unpublished data). Therefore, some eggs contained embryos which were measured using the same method employed to determine egg size.

In this second experiment the hatching rates of the clutches were also estimated. These hatching rates were determined 10 weeks after egg laying. Only empty eggs with open or removed operculum were considered as hatched (Swiderski, 1994).

\section{RESULTS}

\section{Non-experimental cultures}

When worms were alone in a tube, egg size was strongly correlated with the size of the parent: larger cestodes produced larger eggs (Fig. 1 A). Such a sizecorrelated egg production could not be observed in cestodes that were allowed to reproduce in pairs (Fig. 1 B). These 2 correlations were significantly different between the 2 modes of reproduction (see comparison between correlation coefficients in Fig. 1).

However, these non-experimental cultures were started at 6 different times of the year with different numbers of 1-worm or 2-worm cultures each (1worm or 2 -worm cultures at each date: 7:4; 4:5; $0: 13 ; 8: 0 ; 12: 0 ; 9: 0)$. The six sets of in vitro cultures differed also in several other potentially important variables, e.g. the length of the donor fish (ANOVA, $F_{5}=4.82, P=0.002$ ), the parasite index (i.e. the weight of the parasites/net fish weight, ANOVA, $\left.\mathrm{F}_{5}=12 \cdot 66, P<0.001\right)$, the average worm weight (ANOVA, $\mathrm{F}_{5}=28.2, P<0.001$ ), and the average egg size (ANOVA, $\mathrm{F}_{5}=6 \cdot 3, P<0.001$ ). Furthermore, cestodes in pairs were, on average, much smaller than cestodes that were put alone in a tube ( $t=7 \cdot 06, P<0 \cdot 001$; see Fig. $1 \mathrm{~A}$ and $\mathrm{B})$.

\section{Experimental cultures}

First experiment. Larger cestodes again produced larger eggs when kept alone for reproduction (Fig. $1 \mathrm{C})$ but not so when kept in pairs (Fig. 1 D). These 


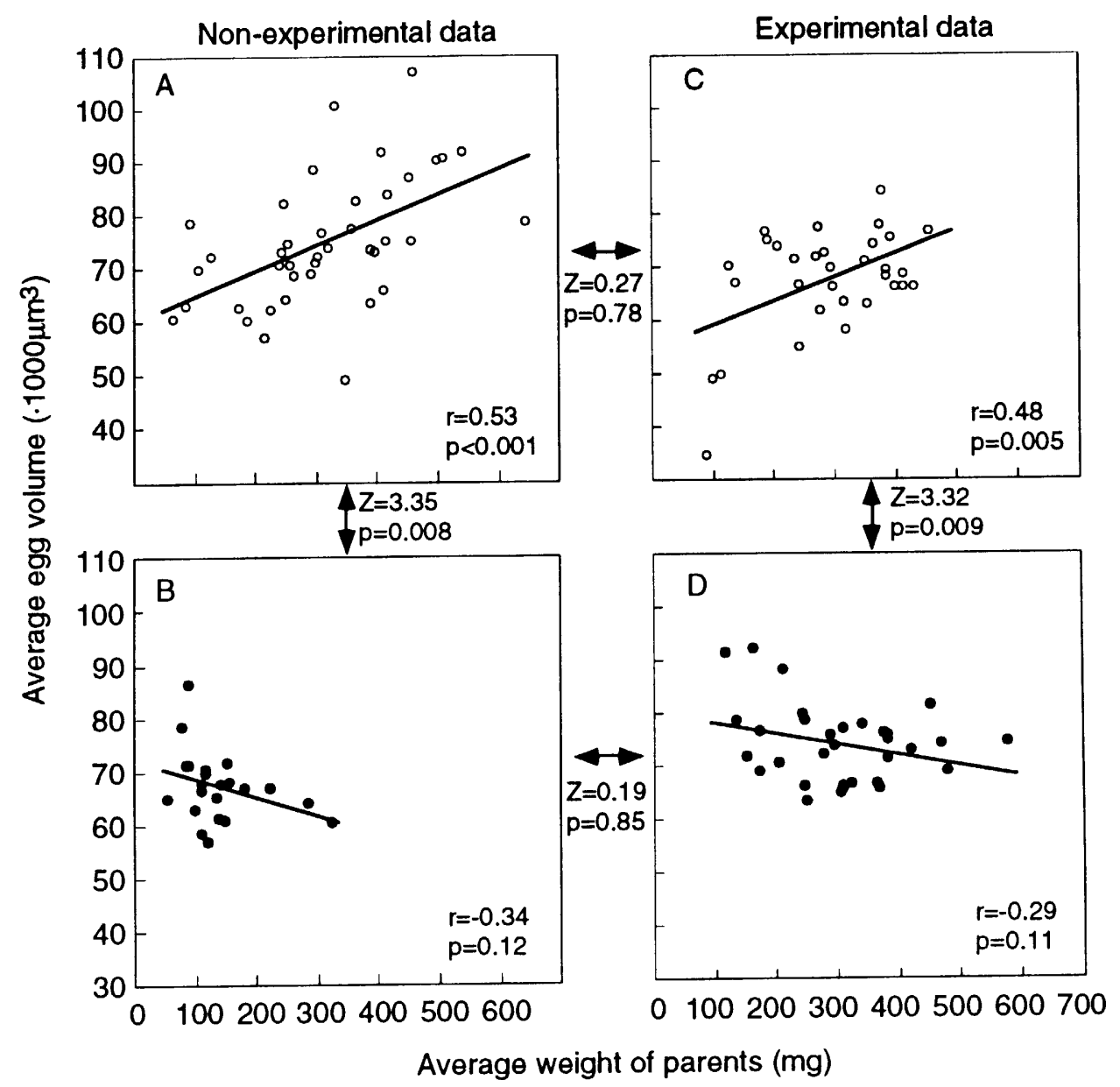

Fig. 1. (A and B) First observations (non-experimental data), and (C and D) experimental data (from Exp. 1): average egg volume/clutch produced during the first 3 days in comparison with the average parental weight (= plerocercoid weight) of Schistocephalus solidus that reproduced alone ( $\bigcirc$; A, C) or in pairs $(\boldsymbol{O}$; B, D). Pearson's correlation coefficients $(r)$ and $P$ values are given within the graphs, comparisons between 2 correlation coefficients $(r)$ each are given between the graphs ( $Z$-tests, see Kleinbaum et al. (1988) p. 92). All $P$ values are 2 -tailed.

2 correlations were again significantly different between the 2 modes of reproduction (see comparison between correlation coefficients in Fig. 1). The first non-experimental observations and the experimental cultures did not significantly differ in the analogous correlations (see $\mathrm{Z}$ - and $\mathrm{P}$-values in Fig. 1). In the experimental cultures a number of additional differences between 1 -worm and 2-worm cultures were found: egg size, egg number and total egg mass production of $S$. solidus depended on whether these hermaphrodites were kept alone or in pairs: although the cestodes in the 2 reproductive conditions did not differ in size (Fig. 2A), cestodes that were allowed to mate with a partner produced on average larger eggs (Fig. 2 B) but isolated cestodes produced more eggs (Fig. 2C) and on average a larger total egg mass (Fig. 2D).

The correlations between egg number and parent weight were not significantly different between cestodes kept alone or in pairs (alone: $r=0 \cdot 47, P<$ 0.01 ; in pairs: $r=0.12, \quad P>0.05$; comparison between correlation coefficients: $Z=1 \cdot 46, \quad P>$ 0.05).

Energy density (measured as Joules/mg dry weight) was higher in worms kept in pairs than in worms kept alone (Fig. 3), indicating that more energy-dense material had been used in singles up to that point.

Second experiment. The average weight of plerocercoids put alone or in pairs into the tubes was not significantly different (alone: $278 \cdot 5 \mathrm{mg} \pm 53 \cdot 2$ s.E.; in pairs: $249 \cdot 1 \mathrm{mg} \pm 38 \cdot 5$ s.E.; $n_{1}=n_{2}=9, t=0 \cdot 45, P$ $=0 \cdot 66)$. In this much smaller sample the differences in egg volume and egg number were not statistically significant between the 2 modes of reproduction (2way ANOVAs with repeated measures, between subjects effect: egg volume: $\mathrm{F}=0 \cdot 01$, D.F. $=1, P=$ 0.91; egg number: $\mathrm{F}=0.51$, D.F. $=1, P=0.49$ ). However, egg production was clearly affected by the length of time the cestodes were in the adult stage. The average size of eggs decreased during the incubation period (Fig. 4A; 2-way ANOVA with 

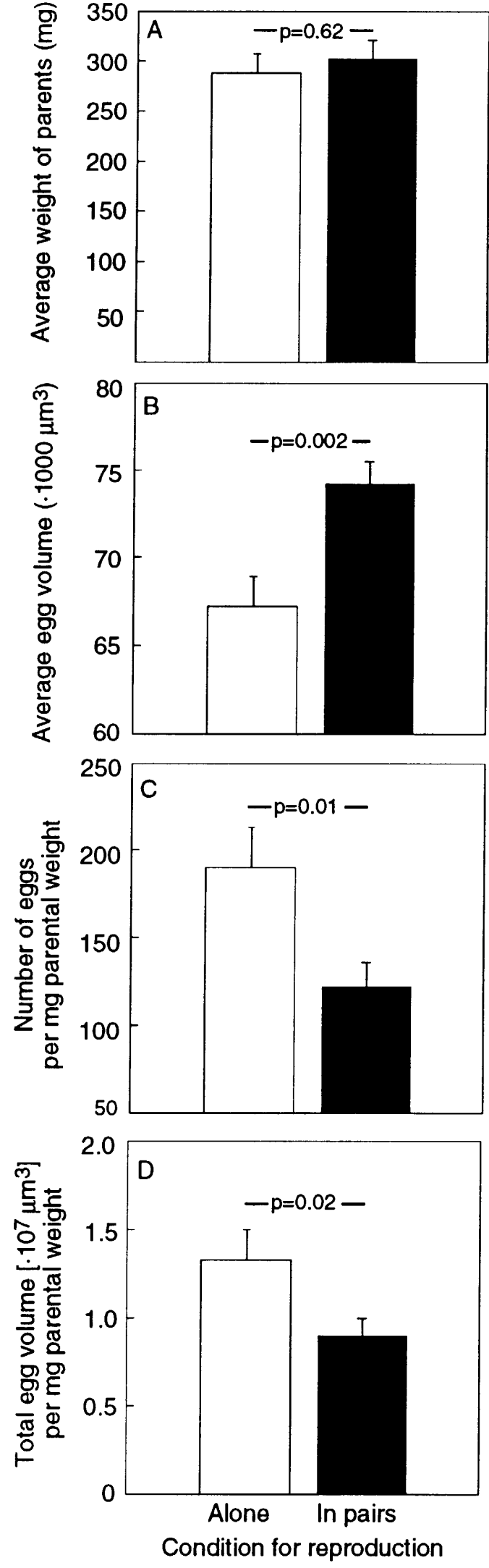

Fig. 2. Exp. 1: egg production of Schistocephalus solidus when kept alone in a tube $(\square)$ or in pairs ( $\square$ ) during the first 3 days of incubation. (A) Average weight of parents, (B) egg volume/clutch, (C) number of eggs/mg weight of parents (plerocercoid weight), and (D) summarized egg volume produced $/ \mathrm{mg}$ weight of

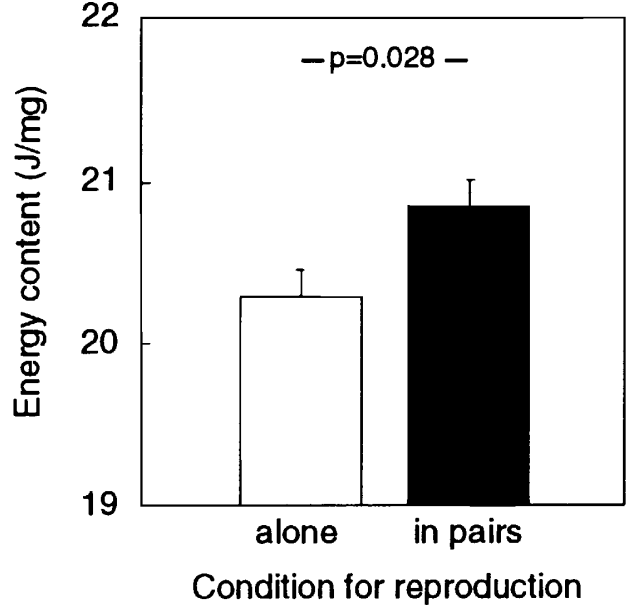

Fig. 3. Exp. 1: energy content as measured in Joules $/ \mathrm{mg}$ dry weight (means \pm s.E.) of a sub-sample $\left(n_{1}=21 ; n_{2}=\right.$ 22) of the Schistocephalus solidus that were kept alone $(\square)$ or in pairs ( $\square$ ) during the 3 days of incubation. The $P$ value stems from a $t$-test, 2 -tailed.

repeated measures, within subjects effect: effect of day: $\mathrm{F}=7.52$, D.F. $=2, P=0.003$; interaction with mode of reproduction: $\mathrm{F}=0.39$, D.F. $=2, P=0.68)$. Furthermore, the average number of eggs per starting weight of parents decreased during the incubation period (Fig. 4B; effect of day: $\mathrm{F}=5 \cdot 70$, D.F. $=2, P=0.008$; interaction: $\mathrm{F}=0.74$, D.F. $=2$, $P=0 \cdot 49)$. The analogous correlation could be observed in the hatching rates 10 weeks after egg laying (Fig. 4C; effect of day: $\mathrm{F}=6 \cdot 84$, D.F. $=2, P=$ 0.005 ; interaction: $\mathrm{F}=2.66$, D.F. $=2, P=0.09$ ).

There was no significant interaction between the 2 conditions of reproduction with respect to the decrease in egg volume, the decrease in egg number, or the decrease in hatching rates over time (see test for interactions above). However, S. solidus that were allowed only to reproduce by selfing achieved over all a lower hatching rate 10 weeks after egg laying than those that could reproduce in pairs (Fig. $5 \mathrm{~A})$.

The size of the embryos which seemed to be fully developed and ready to hatch could be determined in 123 of the eggs measured. Overall, larger eggs produced larger embryos (pooled: $r=0 \cdot 28, n=123$, $P=0 \cdot 002$; eggs from cestodes kept alone: $r=0 \cdot 39, n$ $=43, P=0.01$; eggs from cestodes kept in pairs: $r$ $=0 \cdot 21, n=80, P=0 \cdot 06$ ). The size of the embryos was dependent on the clutch they belonged to and whether or not their parents were alone or in pairs when reproducing (nested ANOVA: effect of reproduction modus: $\mathrm{F}=18 \cdot 90$, D.F. $=1, P<0.001$; effect of family nested in reproduction modus: $\mathrm{F}=$ 5.33 , D.F. $=11, P<0 \cdot 001)$. When egg size was controlled for by using the embryo volume/egg

parents. The figures show the means + s.E.; $P$ values stem from $t$-tests, $n_{1}=n_{2}=32$, two-tailed. 


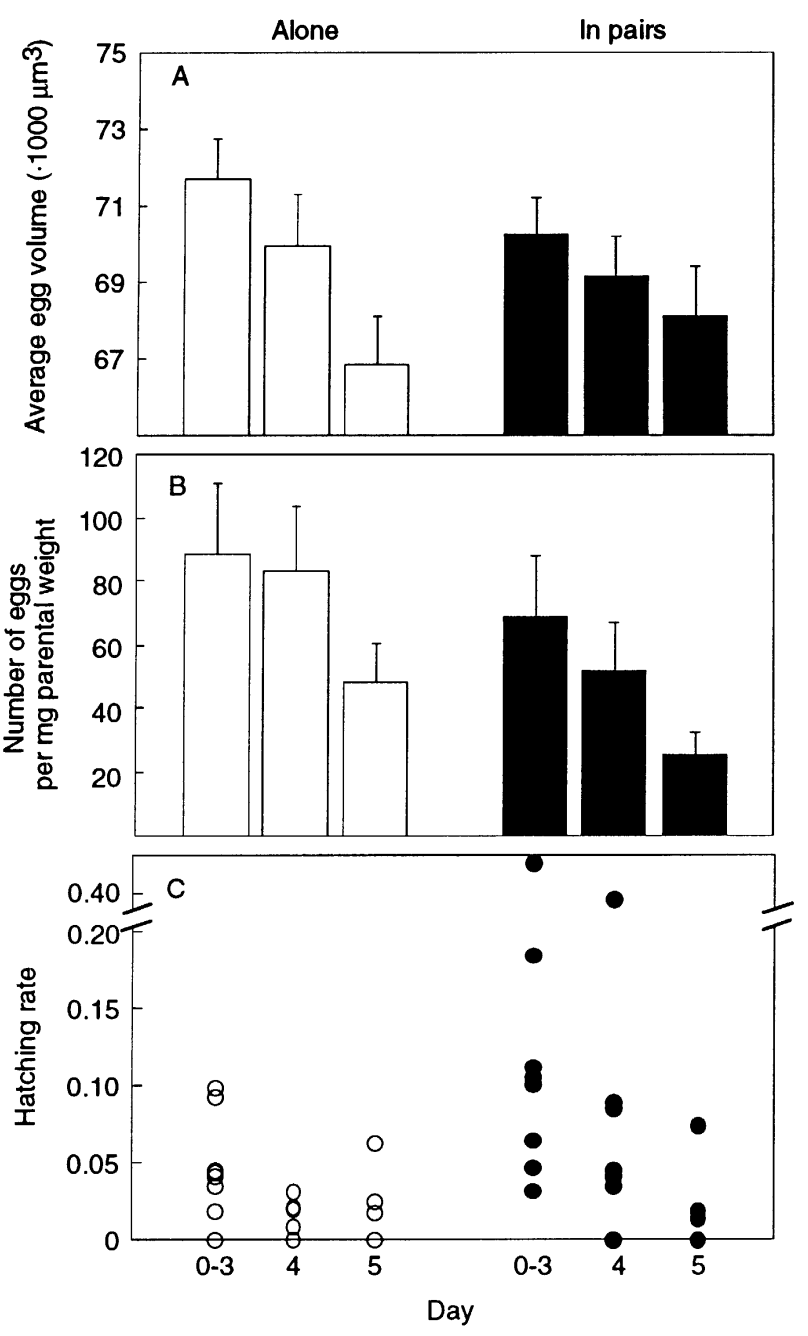

Fig. 4. Second experiment: egg production during the first 3 days, the fourth and the fifth day after start of incubation. (A) Average egg volume (means \pm s.E.), (B) average egg number (means \pm s.E.), and (C) hatching rates (as determined 10 weeks after egg laying) per timeinterval and clutch of Schistocephalus solidus kept alone $(\square)$ or in pairs for reproduction ( $\square$ ). Note than in (C) the scale is not continuous. See text for statistics.

volume as the dependent variable, cestodes reproducing alone were found to produce relatively smaller embryos than cestodes that reproduced in pairs (Fig. 5B; nested ANOVA: effect of reproduction modus: $\mathrm{F}=12.07$, D.F. $=1, \quad P<0.001$; effect of family nested in reproduction modus: $\mathrm{F}=$ $3 \cdot 84$, D.F. $=11, P<0.001)$. The relative embryo size did not correlate significantly with the day of egg removal $(\mathrm{F}=0.61$, D.F. $=2, P=0.55)$, and there was no significant interaction between day of egg removal and reproductive modus in this respect $(\mathrm{F}=1 \cdot 83$, D.F. $=2, P=0 \cdot 16)$.

\section{DISCUSSION}

\section{Cestode reproduction in 2 social situations}

The present study provides the first evidence for strategic egg production in $S$. solidus. The repro-

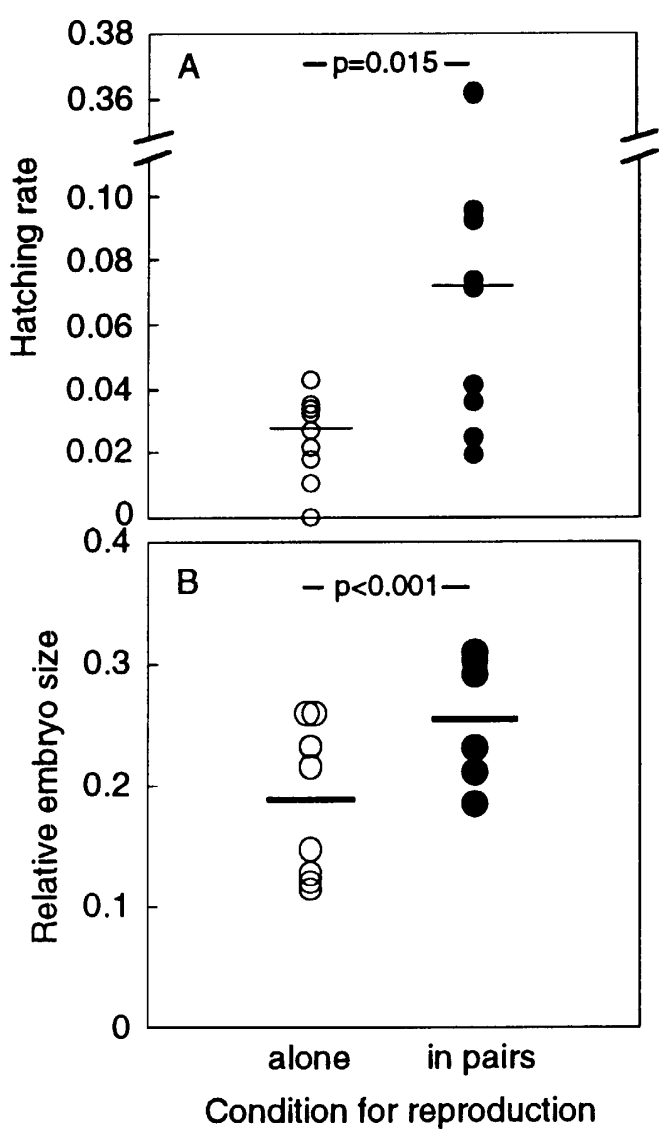

Fig. 5. Second experiment. (A) The overall hatching rates/parent(s) kept alone or in pairs during reproduction, determined for 10 -week-old eggs. The horizontal lines indicate the medians. Note that the scale is not continuous. The $P$ value stems from a Mann-Whitney $U$-test, $n_{1}=n_{2}=9, U=13$, two-tailed. (B) Embryo size relative to egg volume in comparison to the condition for reproduction. Each point represents the average of a clutch, the horizontal line gives the means. The $P$ value stems from a nested ANOVA (see text).

ductive output was found to differ greatly between cestodes kept alone or in pairs for reproduction. Individuals that were kept alone produced smaller eggs but more of them than those kept in pairs. Overall, isolated worms showed a higher egg mass output within the first 3 days of reproduction than worms kept in pairs. This was also reflected in the energy density of worm tissue as determined by micro-bomb calorimetry. It appeared that $S$. solidus kept alone used up more of their resources during the first three days of reproduction. These first 3 days seemed to be the most important ones, since eggs that were produced later were increasingly smaller, fewer in number, and had lower hatching rates - in both reproductive conditions set by us. The decline in egg number observed here corresponds to the observations of Tierney \& Crompton (1992) who had used chickens as experimental host.

When alone, egg production correlated with parent size, i.e. larger worms produced larger eggs. 
Such a size-dependent egg production could not be observed in cestodes kept in pairs. Controlling for a number of potentially confounding variables did not appear to have an influence on these correlations: non-experimental and experimental data look very similar in this respect.

Besides the obvious advantages of replacing the bird host by an in vitro system, a negative consequence of this technique is that it could influence the worms' reproduction. A pair of worms may release and accumulate more harmful metabolites in the culture medium than a single worm. To control for this potential problem, a culture bottle (of the experimental cultures) contained 2 tubes each, 1 tube with a single worm and 1 tube with a pair, i.e. the 2 social conditions shared the same medium conditions. Moreover, relatively large amounts of medium were used here $(250 \mathrm{ml}$ for the nonexperimental cultures and $500 \mathrm{ml}$ for the experimental cultures) with an effective buffer so that the $\mathrm{pH}$ never dropped under 7 as assessed by the colour of the added $\mathrm{pH}$ indicator. Another potential problem may be that the worms could have taken up glucose from the culture medium (Hopkins, 1950). However, the difference in energy contents of paired and single worms as observed in the present study cannot simply be explained by different intake of glucose because the energy content corresponds with total egg volume produced during the incubation period. Therefore, the many observed differences in egg production of singly kept $S$. solidus and paired ones are hardly explained by potential influences of the in vitro system. However, it is still possible that the dynamics of spawning and the total egg output of S. solidus is different in the in vitro system than in the bird host. Potential host responses to infection are difficult to simulate. It actually appears that $S$. solidus survives better in the artificial system since natural host responses may mostly be detrimental to the parasite (e.g. excretion before the end of reproduction as observed by Tierney \& Crompton (1992)).

In the following, the findings of this study are discussed in 4 different but non-exclusive evolutionary frameworks.

\section{Local mate competition adjusted for hermaphrodites}

Maturation of the gonads of S. solidus is known to take place within the first hours after infection of the bird (Smyth, 1946, 1950; Tierney \& Crompton, 1992). If the cestodes have the possibility to regulate their relative investment into male and female reproductive tissues (which is still to be measured, e.g. with histological methods), then one would predict from the local mate competition theory (Hamilton, 1967) adjusted for hermaphrodites (Charlesworth \& Charlesworth, 1981; Charnov, 1982; Petersen, 1990 a) that the cestodes react to the social situation they find themselves in the bird's gut and invest in their reproductive organs accordingly. If alone, a cestode is expected to produce comparatively few sperm - as much as it needs to ensure fertilization of its own eggs. Multiple infection of a bird's gut may, however, result in multiple mating between the cestodes (Nollen, 1983) and, as a likely consequence, in sperm competition (e.g. Michiels, 1998). This can even be expected in situations where only 2 cestodes are interacting with each other. Many parasitic helminths are not completely outbreeding, often some of the sperm found in the receptaculum seminis are their own sperm (reviewed by Nollen, 1983; Trouvé et al. 1996; Nollen, 1997). This probably counterintuitive finding may be explained by the specific costs and benefits of selfing and outbreeding (Jarne \& Charlesworth, 1993; Taylor \& Getz, 1994). In species where individuals are often forced to reproduce by selfing (e.g. in $S$. solidus when alone in the final host during the short period of reproduction), inbred fitness is expected to be comparatively higher than inbred fitness in normally outbreeding species (Jarne \& Charlesworth, 1993). Therefore, it may pay for such parasites to mix selfing and outcrossing even if they are reproducing in pairs (Nollen, 1983) (the existence of a sperm storage organ, the receptaculum seminis (Smyth, 1950), may further increase sperm competition). This may force cross-fertilizing cestodes to invest relatively more into sperm-producing organs at the expense of reduced egg production. It is therefore possible that cestodes which are reproducing alone invest relatively more into their female gonadal tissue and less into male gonadal tissue than cestodes that have the possibility to reproduce by cross-fertilization. This may manifest itself in the amount of total egg mass produced. The cestodes in the present study differed in their overall output of egg mass in the expected direction. However, the rate of allofecondation versus autofecondation is not yet known for this species.

Jarne et al. (1991) found in a snail (Bulinus globosus) that outcrossed clutches were significantly larger than selfed clutches. This is no contradiction to our results, since $S$. solidus differs in a very important aspect from snails or other internally fertilizing hermaphrodites: life-time reproduction of this cestode takes place within few days (Tierney $\&$ Crompton, 1992; Schärer \& Wedekind, unpublished observations). Snails like B. globosus reproduce over a longer period and may therefore be selected for balancing the value of current reproduction to future reproduction. Analogous to the strategy that parasitized snails appear to play (Minchella \& Loverde, 1981; Minchella, 1985), snails that are only allowed to produce by selfing may spare resources for reproduction later in life.

Local mate competition theory can potentially explain the differences in total egg masses observed 
here, but it cannot, for itself, explain a number of further results of this study (e.g. the differences in energy contents of the cestodes, the different egg sizes in the 2 reproductive modi, or the smaller embryos per given egg size of worms reproducing alone).

\section{The hermaphrodite's dilemma}

In situations where several cestodes find themselves in a bird's gut, the individual worms may face a social dilemma situation. This is to be expected since they are simultaneous hermaphrodites, and the investment into each individual offspring is most likely higher for the female part than for the male part. This situation has been called 'the hermaphrodite's dilemma' (Leonard, 1990). Leonard has formulated the hermaphrodite's dilemma in a very general way, summarizing a number of more specified game situations (like e.g. the 'Prisoner's Dilemma', or the 'Chicken Game'). Which game is being played and what the solution of the dilemma, i.e. the players' game strategy, looks like depends very much on a number of characteristics of the dilemma. Important parameters are the pay-off matrices of the 2 players interacting, whether these matrices are the same for both players and whether and how the pay-off matrices change over time. Furthermore, the nature of the interaction (e.g. sequential or alternating), the number of iterations of interactions and how well this number could be estimated by the players is expected to play a crucial role, too. Since the pay-off matrix is not yet known for any particular moment in any given pairing of $S$. solidus (and we do not know of any hermaphrodite species for which it is known), it is at the moment very difficult to make detailed predictions for the worm's mating behaviour on this theoretical basis. At least, the dilemma situation could result in different reproductive strategies that manifested themselves in the rate of energy and resource use/time unit, and in the relative investment/egg measured as egg size, embryo size and hatching rate. The observed differences in these important variables between worms that were kept alone (no social dilemma) and those kept in pairs (social dilemma possible) could reveal game strategies. Players in the hermaphrodite's dilemma may benefit from playing attractive female roles to be allowed to give sperm to their mate. Approaching the end of such a game is expected to lead to more uncooperative game strategies (Axelrod \& Hamilton, 1981). This could be revealed by the decreasing egg size at the fourth and fifth day of incubation.

Our observation that cestodes in pairs produced less egg mass within the first 3 days of incubation could also be interpreted in this framework. If the cestodes face a social dilemma situation they are likely to pay a cost for finding an appropriate game strategy. This cost could be a slightly delayed egg production. Our calorimetric measurements seem to confirm that reproduction was delayed in potential outbreeders.

\section{Bet-hedging}

A next non-exclusive alternative explanation of some of our findings may be spreading the risk of poor development on more but smaller offspring. The main cost of selfing may be genetic deficiencies due to inbreeding depression (Charlesworth \& Charlesworth, 1987; Jarne \& Charlesworth, 1993). This is expected to manifest itself in poorer development of the embryos and a lower hatching rate. Since these genetic deficiencies may be expressed independently of egg size, parents that are forced to reproduce alone could react to this problem by spreading the risk of genetically disturbed development on more but smaller eggs, instead of producing fewer eggs with large resource supply of which many fail to develop because of genetic deficiencies. Whether this explanation holds depends on the relative cost of producing more eggs but smaller ones compared to the benefit of spreading the risk of disturbed development due to inbreeding depression over more offspring. Such data are not available at the moment.

The present findings do not allow quantitative estimation of inbreeding depression, although lower hatching rates in $S$. solidus reproducing alone were found and this suggests at least that inbreeding depression exists. Since there existed a correlation between hatching rate and size of eggs produced by the same parents but during different time-periods, the possibility cannot be excluded that egg size or embryo size differences between clutches produced the observed differences in hatching success. Furthermore, our measure of hatching rate may be a poor one since it cannot be excluded that some eggs would have hatched after the time at which we measured hatching rates (10 weeks after egg laying). Moreover, artefacts with respect to hatching cannot be excluded. To measure inbreeding depression in $S$. solidus will require separation of the reproductive strategies of the cestodes from consequences of genetic defects. This is, in general, a potential problem of measurements of inbreeding depression in many other species - a problem which has also often been overlooked (but see Doums, Delay \& Jarne, 1994; Vernon, 1995). In many studies, inbreeding depression may be overestimated due to the potential of strategic reproduction.

\section{Sib-competition}

Eggs of $S$. solidus sometimes stick to the material they lie on (own observations). Therefore, the eggs of a clutch may under certain conditions stay close 
until hatching, and at the time of hatching individual copepods may take up several coracidia of the same clutch. Larvae that stem from a parent that reproduced by selfing are closely related and therefore expected to exploit the resources of a common host individual in a more cooperative and economic manner than non-related parasites would (Hamilton, 1964; Frank, 1992, 1996). Outbred S. solidus may face larger competition in the host due to smaller relation coefficients within multiple infections (Hamilton, 1964; Frank, 1992, 1996). If the size of the coracidia, i.e. the starting point of growth of $S$. solidus in its first intermediate hosts, is an important variable influencing the outcome of intra-host competition between different individual larvae, outbreeding S. solidus could produce larger eggs with larger embryos to give their offspring better competitive abilities. A necessary pre-requisite for this scenario to be likely is that producing bigger eggs bears some extra cost to the parents.

$S$. solidus is able to adjust a number of crucial parameters of its reproduction according to the social situation in which it finds itself in the final host. This phenotypic plasticity in reproduction of a simultaneous hermaphrodite could be there to achieve a number of different goals, e.g. (1) optimizing sex allocation (local mate competition theory adjusted for simultaneous hermaphrodites), (2) achieving cooperation or taking advantage of mates (hermaphrodite's dilemma), (3) spreading the risk of genetically disturbed embryo development due to inbreeding depression (bet-hedging), or (4) optimizing larval size with respect to the level of competition expected in the first intermediate host and with respect to egg size-dependent parental costs. These concepts make many similar predictions and are therefore still non-exclusive possible explanations of our findings. This study demonstrates that cestodes like $S$. solidus can be suitable model species to test these evolutionary ideas and relate them to parasite-host interactions.

We thank Nathalie Treichel, Barbara Streb, Silvia López, Julian Rauch, Rolf Eggler and Andreas Rüetschi for technical assistance. Thanks also to Uwe Conradt (Düsseldorf, Germany) and Günther Schmahl (Bochum, Germany) for their help when catching the infected fish; Thomas Seebeck for access to the lyophilizator; Ingolf Lamprecht (Berlin, Germany) for access to his calorimeter and Mrs Welge for help with the sample analyses; Nico Michiels for access to unpublished manuscripts; and Manfred Milinski, Nico Michiels, Mira Christen, Kati Turi Nagy and the anonymous referees for discussion or critical comments on the manuscript. This project was supported by the Swiss National Science Foundation (grant number 31-45'733.95).

\section{REFERENCES}

AXElrod, R. \& Hamilton, W. D. (1981). The evolution of cooperation. Science 211, 1390-1396.
BRUNET, J. (1992). Sex allocation in hermaphroditic plants. Trends in Ecology and Evolution 7, 79-84.

CHARlESWORTH, D. \& CHARLESWORTH, B. (1981).

Allocation of resources to male and female functions in hermaphrodites. Biological Fournal of the Linean Society 15, 57-74.

CHARLESWORTH, D. \& CHARLESWORTH, B. (1987). Inbreeding depression and its evolutionary consequences. Annual Review of Ecology and Systematics 18, 237-268.

Charnov, E. L. (1982). The Theory of Sex Allocation. Princeton University Press, Princeton.

Clarke, A. S. (1954). Studies on the life cycle of the pseudophyllidean cestode Schistocephalus solidus. Proceedings of the Zoological Society of London 124, 257-304.

DE VISSER, J. A. G. M., TER MAAT, A. \& ZONNEVELD, C. (1994). Energy budgets and reproductive allocation in the simultaneous hermaphrodite pond snail, Lymnaea stagnalis (L.): a trade-off between male and female function. American Naturalist 144, 861-867.

DOUMS, C., DELAY, B. \& JARNE, P. (1994). A problem with the estimate of self-fertilization depression in the hermaphrodite freshwater snail Bulinus truncatus: the effect of grouping. Evolution 48, 498-504.

DOUMS, C., VIARD, F., PERNOT, A.-F., DELAY, B. \& JARNE, P. (1996). Inbreeding depression, neutral polymorphism, and copulatory behavior in freshwater snails: a selffertilization syndrome. Evolution 50, 1908-1918.

FISCHER, E. A. (1981). Sexual allocation in a simultaneously hermaphroditic coral reef fish. American Naturalist 117, 64-82.

FRANK, S. A. (1992). A kin selection model for the evolution of virulence. Proceedings of the Royal Society of London, B 250, 195-197.

FRANK, s. A. (1996). Models of parasite virulence. The Quarterly Review of Biology 71, 37-77.

hamilton, w. D. (1964). The genetical evolution of social behaviour. Fournal of Theoretical Biology 7, $1-16$.

HAMilton, W. D. (1967). Extraordinary sex ratios. Science 156, 477-488.

HOPKINS, C. A. (1950). Studies on cestode metabolism. I. Glycogen metabolism in Schistocephalus solidus in vivo. Fournal of Parasitology 36, 384-390.

JARNE, P. \& CHARLESWORTH, D. (1993). The evolution of the selfing rate in functionally hermaphrodite plants and animals. Annual Reviews in Ecology and Systematics 24, 441-466.

JARNE, P. \& CHARLESWORTH, D. (1996). Hermes meets aphrodite: an animal perspective. Trends in Ecology and Evolution 11, 105-107.

JaRne, P., Finot, L., DElay, B. \& Thaler, L. (1991). Selffertilization versus cross-fertilization in the hermaphroditic freshwater snail Bulinus globosus. Evolution 45, 1136-1146.

JOHNSTON, M. O., DAs, B. \& HOEH, W. R. (1998). Negative correlation between male allocation and rate of selffertilization in a hermaphroditic animal. Proceedings of the National Academy of Sciences, USA 95, 617-620.

KLEINBAuM, D. C., KUPPER, L. L. \& MUller, K. E. (1988). Applied Regression Analysis and Other Multivariate Methods. Boston, PWS-Kent Publishing Company. 
LEONARD, J. L. (1990). The hermaphrodite's dilemma. Fournal of Theoretical Biology 147, 361-372.

LEONARD, J. L. (1993). Sexual conflict in simultaneous hermaphrodites: evidence from serranid fishes. Environmental Biology of Fishes 36, 135-148.

LEONARD, J. L. \& Lukowiak, K. (1991). Sexual and the simultaneous hermaphrodites: testing models of male-female conflict in a sea slug. Animal Behaviour 41, 255-266.

MARY, C. M. S. (1994). Sex allocation in a simultaneous hermaphrodite, the blue banded goby (Lythrypnus dalli): the effects of body size and behavioral gender and the consequences for reproduction. Behavioral Ecology 5, 304-313.

Michiels, N. (1998). Mating conflicts and sperm competition in simultaneous hermaphrodites. In Sperm Competition and Sexual Selection, (ed. Birkhead, T. R. \& Møller, A. P.), Academic Press, New York (in the Press).

MINCHELla, D. J. (1985). Host life-history variation in response to parasitism. Parasitology 90, 205-216.

MiNCHELla, D. J. \& LOVERDE, P. T. (1981). A cost of increased early reproductive effort in the snail Biomphalaria glabrata. American Naturalist 118, 876-881.

NOLLEN, P. M. (1983). Pattern of sexual reproduction among parasitic platyhelminths. Parasitology 86, 99-120.

NOLLEN, P. M. (1997). Mating behaviour of Echinostoma caproni and E. trivolvis in concurrent infections in hamsters. International fournal for Parasitology 27, $71-75$.

PARKER, G. A. (1984). Sperm competition and the evolution of animal mating strategies. In Sperm Competition and the Evolution of Animal Mating Systems, (ed. Smith, R. L.), pp. 1-60. Academic Press, New York.

PETERSEN, C. W. (1990a). Sex allocation in simultaneous hermaphrodites: testing local mate competition theory. Lectures on Mathematics in the Life Science 22, 183-205.

PETERSEN, C. W. (1990b). The relationships among population density, individual size, mating tactics, and reproductive success in a hermaphroditic fish, Serranus fasciatus. Behaviour 113, 67-80.

PETERSEN, C. W. \& FISCHER, E. A. (1996). Intraspecific variation in sex allocation in a simultaneous hermaphrodite: the effect of individual size. Evolution 50, 636-645.

Phillipson, J. (1964). A miniature bomb calorimeter for small biological samples. Oikos 15, 130-139.

PREMOLI, M. C., SELla, G. \& BERRA, G. P. (1996). Heritable variation of sex ratio in a polychaete worm. Fournal of Evolutionary Biology 9, 845-854.
RAIMONDi, P. T. \& MARTIN, J. E. (1991). Evidence that mating group size affects allocation of reproductive resources in a simultaneous hermaphrodite. American Naturalist 138, 1206-1217.

SELLA, G. (1990). Sex allocation in the simultaneously hermaphroditic polychaete worm Ophryotrocha diadema. Ecology 71, 27-32.

SMYth, J. D. (1946). Studies of tapeworm physiology. I. The cultivation of Schistocephalus solidus in vitro. Fournal of experimental Biology 23, 47-70.

SMYTH, J. D. (1950). Studies on tapeworm physiology. V. Further observations on the maturation of Schistocephalus solidus (Diphyllobothriidae) under sterile conditions in vitro. Fournal of Parasitology 3, $371-383$.

SMYTH, J. D. (1954). Studies on tapeworm physiology. VII. Fertilization of Schistocephalus solidus in vitro. Experimental Parasitology 3, 64-71.

SWIDERSKI, z. (1994). Origin, differentiation and ultrastructure of egg envelopes surrounding the coracidia of Bothriocephalus clavibothrium (Cestoda, Pseudophyllidea). Acta Parasitologica 39, 73-81.

systat (1992). Statistics, Version 5.2 Edition. SYSTAT Evanston, IL.

TAYLOR, P. D. \& GETZ, W. M. (1994). An inclusive fitness model for the evolutionary advantage of sibmating. Evolutionary Ecology 8, 61-69.

TIERNEY, J. F. \& CROMPTON, D. W. T. (1992). Infectivity of the plerocercoids of Schistocephalus solidus (Cestoda: Ligulidae) and fecundity of the adults in an experimental definitive host, Gallus gallus. Fournal of Parasitology 78, 1049-1054.

Trouvé, S., RENAUd, F., DURAND, P. \& JOURDANE, J. (1996). Selfing and outcrossing in a parasitic hermaphrodite helminth (Trematoda, Echinostomatidae). Heredity 77, 1-8.

VERNON, J. G. (1995). Low reproductive output of isolated, self-fertilizing snails: inbreeding depression or absence of social facilitation. Proceedings of the Royal Society of London, B 259, 131-136.

vREYs, C. \& Michiels, N. K. (1997). Flatworms flatten to size up each other. Proceedings of the Royal Society of London, B 264, 1559-1564.

Wedekind, c. (1997). The infectivity, growth, and virulence of the cestode Schistocephalus solidus in its first intermediate host, the copepod Macrocyclops albidus. Parasitology 115, 317-324.

WEDEKIND, C. \& Milinski, M. (1996). Do three-spined sticklebacks avoid to consume copepods, the first intermediate host of Schistocephalus solidus? - An experimental analysis of behavioural resistance. Parasitology 112, 371-383. 